\title{
Numerical analysis of latent heat storage system with encapsulated phase change material in spherical capsules
} \author{
and Manuel Romero ${ }^{1, *}$ \\ 1 IMDEA Energy Institute, Ramón de la Sagra 3, 28935 Móstoles, Spain \\ ${ }^{2}$ University of Genoa, Genoa, Italy
}

Selvan Bellan ${ }^{1}$, Alice Cordiviola ${ }^{2}$, Stefano Barberis ${ }^{2}$, Alberto Traverso $^{2}$, José González-Aguilar ${ }^{1}$,

\begin{abstract}
Solar energy has been considered as one of the promising solutions to replace the fossil fuels. To generate electricity beyond normal daylight hours, thermal energy storage systems (TES) play a vital role in concentrated solar power (CSP) plants. Thus, a significant focus has been given on the improvement of TES systems from the past few decades. In this study, a numerical model is developed to obtain the detailed heat transfer characteristics of lab-scale latent thermal energy storage system, which consists of molten salt encapsulated spherical capsules and air. The melting process and the corresponding temperature and velocity distributions in every capsule of the system are predicted. The enthalpy-porosity approach is used to model the phase change region. The model is validated with the reported experimental results. Influence of initial condition on the thermal performance of the TES system is predicted.
\end{abstract}

\section{Introduction}

One of the important advantages of CSP technology is the thermal energy storage system, which stores the heat for later use and increases the hours of electricity generation and dispatch ability $[1,2]$. Several methods of storing thermal energy (sensible, latent and thermochemical) for different temperature ranges have been carried out from the past few decades. Latent thermal energy storage (LTES) system with phase change materials (PCM) is considered as one of the attractive methods since it provides high volumetric energy storage density resulting in low capital cost than the sensible heat storage systems $[3,4]$. However, a major disadvantage of LTES is the low heat transfer rate due to high thermal resistance caused by the low thermal conductivity of the PCMs. Various methods have been proposed to enhance the heat transfer rate. Liwu and Khodadadi [5] reviewed the thermal conductivity enhancement of PCMs for thermal energy storage applications. Embedded heat pipes were used by Nithyanandam et al. [6] to enhance the heat transfer between the HTF and the PCM. The PCM was mixed with multi-walled carbon nanotubes [7] and expanded graphite [8] to augment the heat transfer properties. A design for heat transfer enhancement using radially finned tubes was proposed by DLR [9]. Micro encapsulation method was used by Platte et al. [10].

\footnotetext{
* e-mail: manuel.romero@imdea.org
}

A promising approach to overcome this problem is to increase the heat transfer area between the PCM domain and the heat transfer fluid. Since a large amount of heat transfer surface area can be obtained in a small volume, packed bed TES systems have been developed in recent times [11].

Liu et al. [12] reviewed the storage materials and thermal performance enhancement techniques for high temperature thermal storage systems. Suitable phase change materials for high temperature processes have been investigated since the LTES system with PCM has been identified as a promising low-cost system for CSP plants; molten salt has been found as promising material to be used as the PCM for high temperature storage systems, range from $100{ }^{\circ} \mathrm{C}$ to above $600{ }^{\circ} \mathrm{C}$. Thus, numerical models have been developed to investigate the thermal performance characteristics of the LTES system with molten salt as the PCM $[2,11,13-17]$. The main aim of this investigation is to numerically analyze the charging behavior of latent heat storage system with encapsulated phase change material in spherical capsules. In order to study the fluid flow and melting process inside every capsule of the system and to predict the natural convection effect, a numerical model is developed. Molten salt is used as the PCM; the shell of the capsule is made up of polymer. Air is used as heat transfer fluid (HTF). The processes occur in solid PCM due to conduction, liquid PCM due to convection and the solid-fluid interface movements are predicted in each capsule of the system. The thermal performance of the system is studied for various operating parameters. 


\section{Modelling of latent thermal energy storage system}

Schematic of the lab-scale cylindrical thermal energy storage system filled with spherical capsules is shown in Figure 1. The height and the radius of the porous bed region are $0.254 \mathrm{~m}$ and $0.127 \mathrm{~m}$ respectively. The tank consists of 770 capsules with an average diameter of $0.0275 \mathrm{~m}$. The void fraction of the tank is 0.354 . The tank is well insulated by $0.1524 \mathrm{~m}$ thickness insulation layer. In order to study the heat transfer characteristics of TES system, a numerical model is developed with the following assumptions:

(1) The flow of molten PCM and HTF is unsteady, incompressible and laminar.

(2) Solid and liquid phases of the PCM are isotropic and homogenous.

(3) The radiation heat transfer is negligible between the capsules.

(4) The melting of the PCM is assumed between 305.6 and $307.6^{\circ} \mathrm{C}$ [18], the temperature dependent liquid phase density is defined as $\rho_{l i q}=\rho_{m} / \beta\left(T-T_{m}\right)+1$. As the PCM volume changes during the phase change process, the elastic deformation takes place in the shell material [19].

(5) The capsules are filled with rhombic packing, as a result, the contact between the capsules is point-to-point; consequently the heat transfer between the capsules is negligible. Therefore, the void space between the capsules is assumed, because the 2D model does not lead to flow through the bed if the capsules contact to each other $[20,21]$.

(6) In packed bed system of spherical capsules, the cross sectional temperature and velocity distributions are uniformly distributed except the near wall region [11,22]. Thus, a unit region close to the axis is considered as shown in Figure 1. Instead of explicitly tracking the liquid-solid front, the enthalpy-porosity method is used to simulate the melting process. Given the above assumptions, the conservation of mass, momentum and energy equations to simulate the flow and heat transfer are;

$$
\begin{aligned}
\frac{\partial \rho}{\partial t}+\nabla \cdot(\rho \vec{V}) & =0 \\
\rho \frac{\partial \vec{V}}{\partial t}+\rho(\vec{V}+. \nabla) \vec{V} & =-\nabla P \\
& +\nabla \cdot\left[\mu\left(\nabla \vec{V}+(\nabla \vec{V})^{T}\right)\right]+\vec{S} \\
\rho C_{p} \frac{\partial T}{\partial t}+\rho C_{p} \vec{V} \cdot \nabla T & =\nabla \cdot(k \nabla T)
\end{aligned}
$$

where $\rho, \vec{V}, P, \mu, k$, and $T$ are density, velocity vector, pressure, dynamic viscosity, thermal conductivity, and temperature respectively. The source term $\vec{S}$ in the momentum equation is not considered in the HTF domain, whereas in the PCM region which is defined as.

$$
\vec{S}=\rho \vec{g}+A(\gamma) \vec{V}
$$

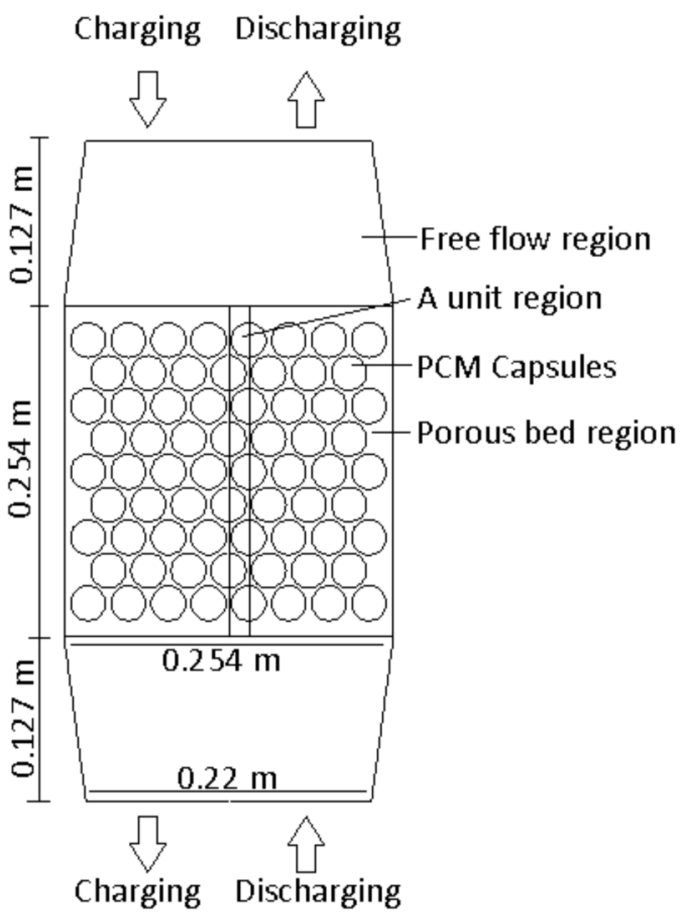

Fig. 1. Schematic of thermal energy storage system.

The second term in the above equation is the Dacry's damping term, which is included to introduce the phase change effect on convection. The porosity function $A(\gamma)$ is defined by Eq. (5)

$$
A(\gamma)=\frac{C(1-\gamma)^{2}}{\gamma^{3}+\varepsilon}
$$

where the computational constant $(\varepsilon)$, and the melting front morphology constant (C) are 0.001 and $105 \mathrm{~kg} / \mathrm{m}^{3} \mathrm{~s}$ respectively. The melt fraction, $\gamma$, at each element of the PCM domain is given by:

$$
\gamma= \begin{cases}0 & \text { if } T<T_{\text {sol }} \\ \frac{T-T_{\text {sol }}}{T_{l i q}-T_{\text {sol }}} & \text { if } T_{\text {sol }} \leq T \leq T_{l i q} \\ 1 & \text { if } T>T_{l i q}\end{cases}
$$

Temperature dependent thermo-physical properties of the PCM are taken from Ref. [11]. The temperature and flow conditions at the inlet and outlet are:

$$
\begin{aligned}
\text { Inlet }: T_{f}=T_{f \text { inlet }}(t), \quad u & =u_{\text {inlet }}, \\
\text { Outlet }: \frac{\partial T}{\partial n}=0, \quad \frac{\partial u}{\partial n} & =0 .
\end{aligned}
$$

The finite element method based commercial code Comsol multiphysics 4.2 is used to solve the governing equations. In order to solve the unsteady fluid flow and heat transfer, the software proposed solver PARDISO is used to solve simultaneously the unknown variables (velocity, temperature and pressure) in the discretized system of equations. 


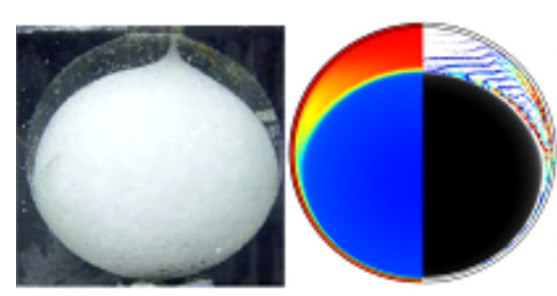

(a) $20 \mathrm{~min}$.
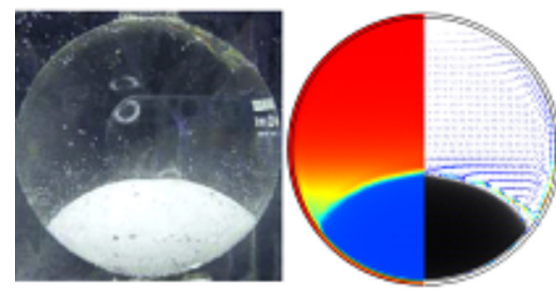

(c) $60 \mathrm{~min}$.
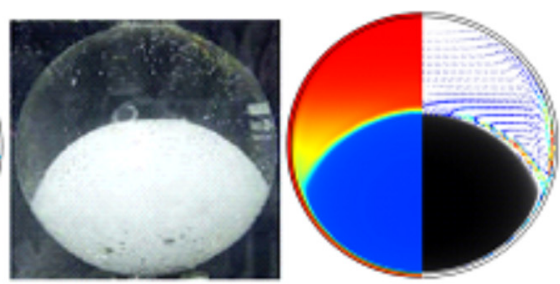

(b) $40 \mathrm{~min}$.
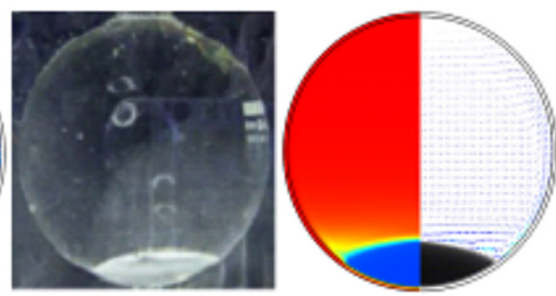

(d) $80 \mathrm{~min}$.

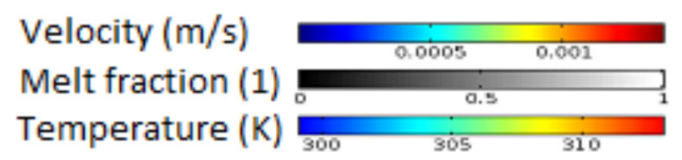

Fig. 2. Experimentally captured and numerically predicted melting front at various stages: (a) $20 \mathrm{~min}$, (b) $40 \mathrm{~min}$, (c) $60 \mathrm{~min}$ and (d) $80 \mathrm{~min}$.

\section{Results and discussion}

In order to validate the developed model, the melting process of $n$-octadecane inside a spherical glass capsule has been simulated and compared with the experimental results [23]. In the experiments, the PCM was filled in a 50.83-mm inner radius capsule and $1.5-\mathrm{mm}$ wall thickness. Initially, the capsule was kept at $1{ }^{\circ} \mathrm{C}$ less than the melting point $\left(29^{\circ} \mathrm{C}\right)$, when the $t>0$ the outer surface of the capsule was fixed at $40^{\circ} \mathrm{C}$. Simulation has been performed for the same conditions. The melting front, temperature and velocity distributions of the capsule during melting are shown in Figure 2 at 20-minute intervals. Experimental photographs of the melting of the PCM inside the capsule are compared with the numerical results. In the simulation results, the left side represents the temperature distribution and the right side represents the melt fraction and the velocity vector. As a good agreement has been found between the numerical and experimental results of single capsule melting process, the same approach has been extended to a high temperature lab scale packed bed system developed by Tanvir et al., [18]. During the charge mode, the blower was connected at the top port of the system; the temperature and flow rate of the HTF were fixed at $286{ }^{\circ} \mathrm{C}$ and $110 \mathrm{~m}^{3} / \mathrm{h}$ respectively. The HTF was circulated through the system until it reached $286^{\circ} \mathrm{C}$ (initial temperature) throughout the tank. In order to carry out the charging process, the temperature of the HTF was fixed at $326^{\circ} \mathrm{C}$ and the charging process was performed and monitored until the temperature of the tank reached $326^{\circ} \mathrm{C}$. Simulation has been carried out for the same conditions. Figure 3 shows the comparison of experimentally measured and numerically predicted charging time of the tank at various axial positions of the bed. The charging

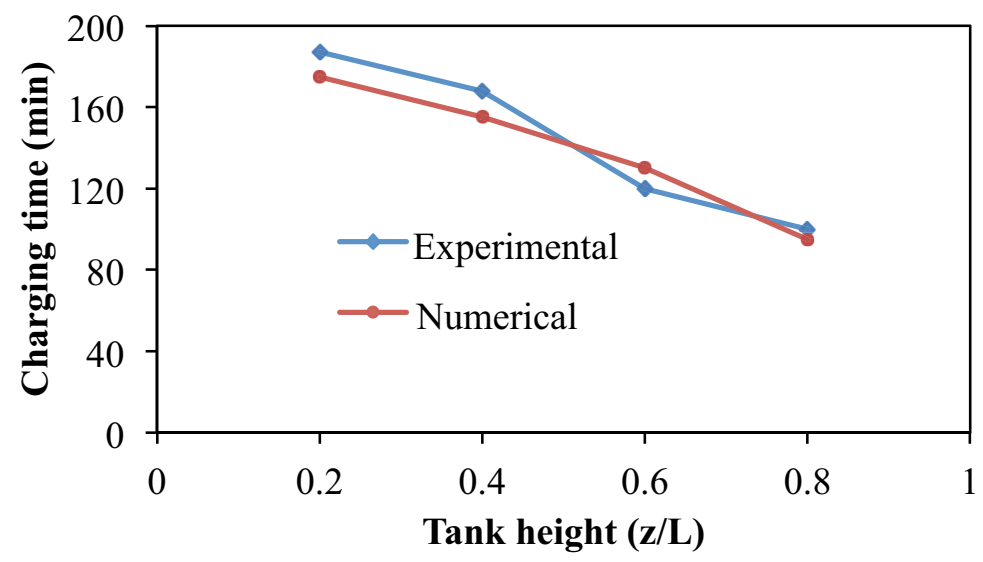

Fig. 3. Charging time of the tank at different height of the bed for various flow rates. 

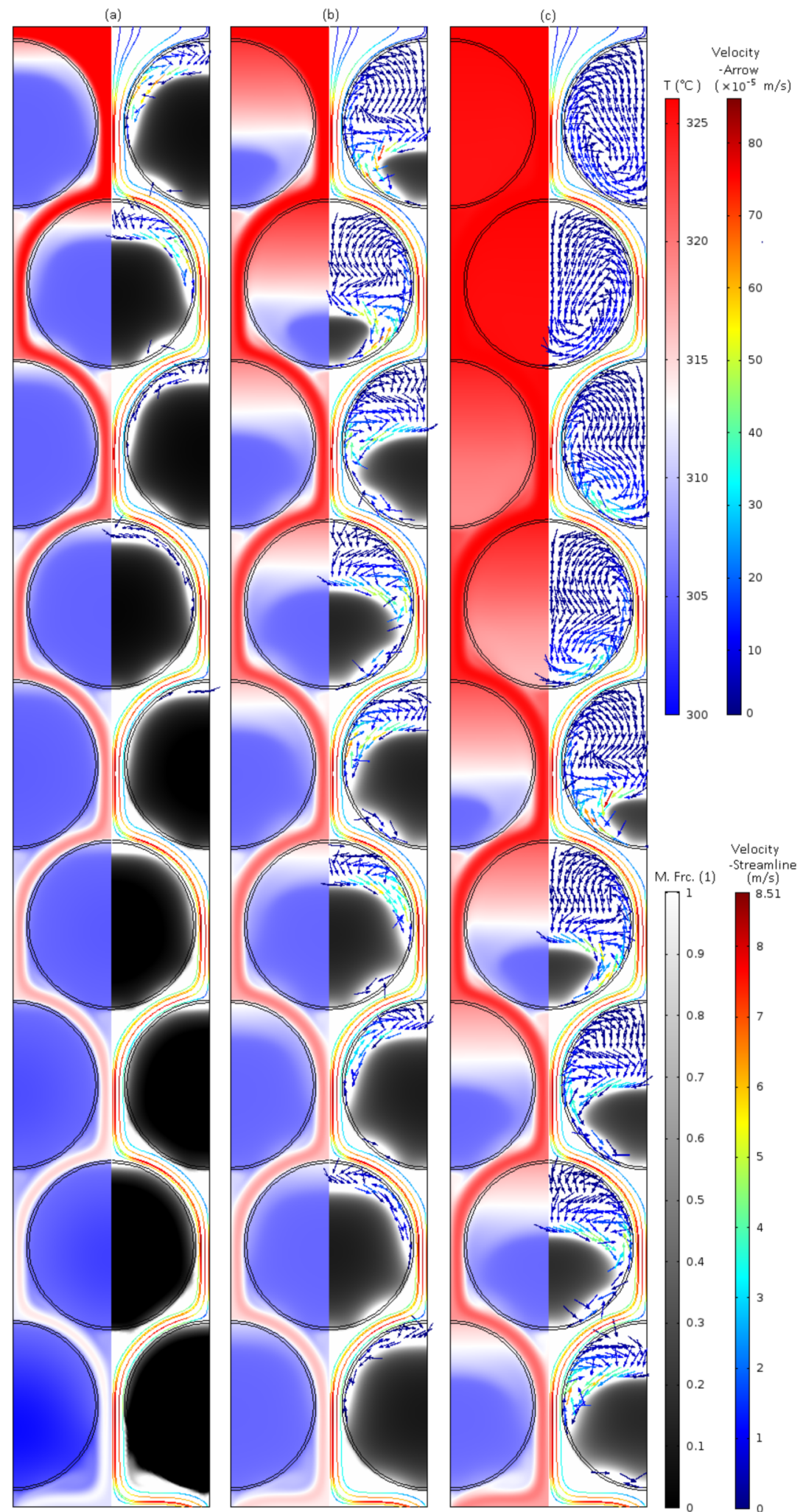

Fig. 4. Temperature, melt fraction and velocity distributions of the tank during charge mode at various stages: (a) 30 min, (b) 60 min, and (c) $90 \mathrm{~min}$.

time represents the time required to reach the inlet HTF temperature at the given height of the packed bed. As can be seen in the figure, the simulation results are comparable with experimental results.
In order to investigate the effect of initial condition of the bed on thermal performance of the system, inlet temperature and the flow rate of the HTF were fixed at $326^{\circ} \mathrm{C}$ and $110 \mathrm{~m}^{3} / \mathrm{h}$ respectively, and simulations were 


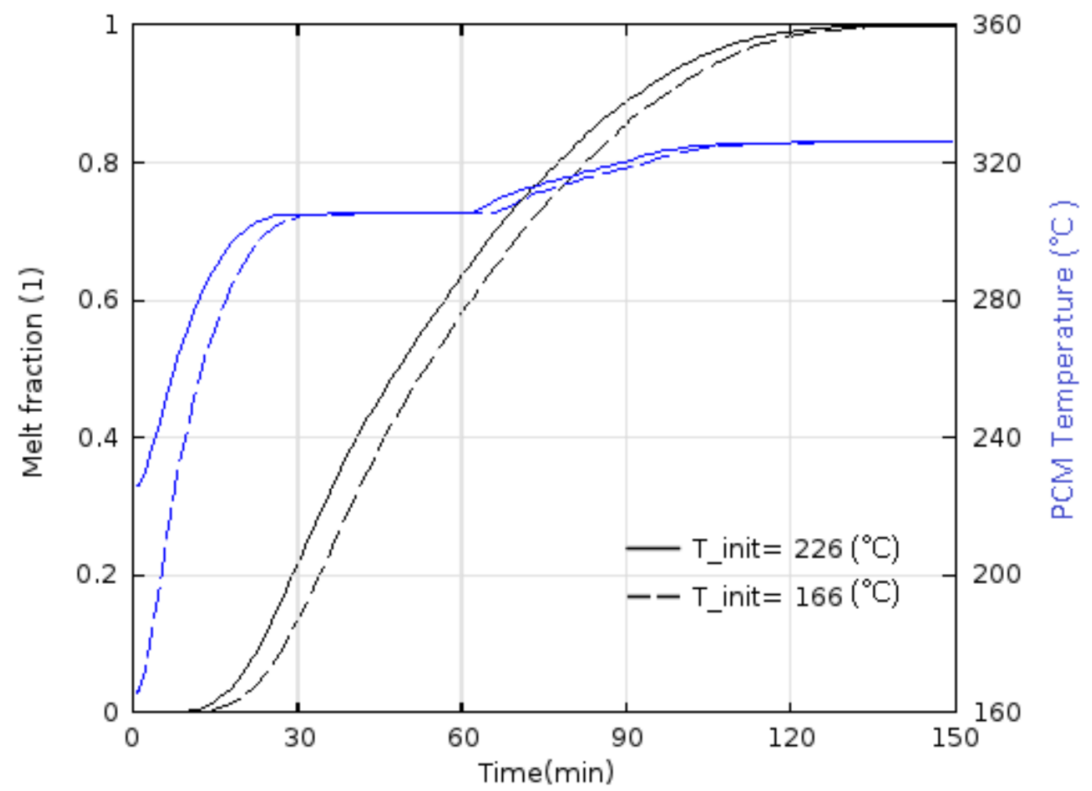

Fig. 5. PCM temperature at $z / L=0.6$ and melt fraction of the bed for two different initial conditions as a function of time.

carried out for two different initial conditions; 166 and $226^{\circ} \mathrm{C}$. The detailed heat transfer, melting and natural convection process at each capsule of the system is shown in Figure 4, which shows the temperature distribution, melt fraction and velocity distribution of the tank during charge mode at various stages; (a) $30 \mathrm{~min}$; (b) $60 \mathrm{~min}$; and (c) $90 \mathrm{~min}$ (for $T_{\text {init }}=226{ }^{\circ} \mathrm{C}$ ). In each chart, the left side refers the temperature distribution of the PCM and HTF domains. The right side of the PCM domain refers the melt fraction (black and white gradient in PCM domain) and velocity vector of the flow inside the capsule. The velocity of the HTF is shown in the right side of the void region as stream lines. It is noticed that the melting process exactly depends on the HTF velocity at the external surface of the shell. As can be seen in the figure, this model enables to obtain more details of the thermo-fluid flow characteristics of the system.

Figure 5 shows the PCM temperature at $z / L=0.6$ and the melt fraction of the bed as a function of time for two different initial conditions. A significant difference between the temperatures is observed only during the initial stage, until it reaches the melting temperature. Then the difference is diluted as shown in the figure. Consequently, there is no significant difference is noticed in the melt fraction and the complete melting time. When reducing the initial temperature of the bed around $26 \%$, the time to obtain fully molten state of the bed increased about $4 \%$.

\section{Conclusion}

A numerical model has been developed to study the detailed analysis of heat transfer and fluid flow of each capsule of the thermal energy storage system in high temperature range. The model has been validated with the reported experimental results. The detailed results of melting process and the corresponding temperature and velocity distributions have been predicted and the influence of initial condition on the thermal performance of the system has been obtained.
This model can be used to obtain detailed heat transfer characteristics of the lab scale TES system and to derive the natural convection correlation for large scale systems.

Authors gratefully acknowledge the Spanish Ministry of Economy and Innovation through Plan Nacional $\mathrm{I}+\mathrm{D}+\mathrm{i}$ project No. ENE2011-29293; "Comunidad de Madrid" and "European Social Fund" for its financial support to the ALCCONES Project through the Programme of Activities between Research Groups (S2013/ MAE-2985). The research leading to these results has received funding from the European Union Seventh Framework Programme (FP7/2007-2013) under grant agreement No. 60983.

\section{References}

1. S. Kuravi, J. Trahan, D.Y. Goswami, M.M. Rahman, E.K. Stefanakos, Prog. Energy Combust. Sci. 39, 285 (2013)

2. W. Ming, X. Chao, H. Ya-Ling, Appl. Energy 121, 184 (2014)

3. S.M. Hasnain, Energy Convers. Manag. 39, 1127 (1998)

4. D. Ibrahim, R. March, Thermal energy storageNew York: Wiley-Interscience (2002)

5. F. Liwu, J.M. Khodadadi, Renew. Sustain. Energy Rev. 15, $24(2011)$

6. K. Nithyanandam, R. Pitchumani, J Heat Mass Transfer 54, 4596 (2011)

7. V. Kumaresan, R. Velraj, S.K. Das, Heat Mass Transfer 48, 1345 (2012)

8. X. Xiao, P. Zhang, M. Li, Energy Convers. Manag. 73, 86 (2013)

9. D. Laing, T. Bauer, N. Breidenbach, B. Hachmann, M. Johnson, Appl. Energy 109, 497 (2013)

10. D. Platte, U. Helbig, R. Houbertz, G. Sextl, Macromol. Mater. Eng. 298, 67 (2013)

11. S. Bellan, J.G. Aguilar, M. Romero, M.M. Rahman, D.Y. Goswami, E. Stefanakos, D. Couling, Appl. Therm. Eng. 71, $481(2014)$

12. M. Liu, W. Saman, F. Bruno, Renew. Sustain. Energy Rev. 16, $2118(2012)$ 
13. K. Nithyanandam, R. Pitchumani, A. Mathur, Appl. Energy 113, 1446 (2014)

14. Y. Zheng, J.L. Barton, K. Tuzl, J.C. Chen, S. Neti, A. Oztekin, W.Z. Misiolek, Sol. Energy 115, 180 (2015)

15. H. Peng, H. Dong, X. Ling, Energy Convers. Manag. 81, 420 (2014)

16. S. Bellan, J.G. Aguilar, M. Romero, M.M. Rahman, D.Y. Goswami, E.K. Stefanakos, Appl. Therm. Eng. 90, 970 (2015)

17. S. Bellan, J.G. Aguilar, M. Romero, M.M. Rahman, D.Y. Goswami, E.K. Stefanakos, Energy Proc. 69, 758 (2015)

18. T.E. Alam, S. Bellan, D. Jaspreet, D.Y. Goswami, M.M. Rahman, E.K. Stefanakos, J.G. Aguilar, M. Romero, in Proc. of the EuroSun conference, 16-19 September, Aix-les Bains, France (2014)
19. T. E. Alam, S.D. Jaspreet, D.Y. Goswami, E.K. Stefanakos, Appl. Energy 154, 92 (2015)

20. S. Bellan, J.G. Aguilar, M. Romero, M.M. Rahman, D.Y. Goswami, E.K. Stefanakos, in Proc. of the ASME 8th International Conference on Energy Sustainability, BostonUSA, (2014). DOI:10.1115/ES2014-6382

21. S. Bellan, J.G. Aguilar, A.R. Archibold, M. Romero, M.M. Rahman, D.Y. Goswami, E.K. Stefanakos, Energy Proc. 57, 672 (2014)

22. X. Chao, W. Zhifeng, H. Yaling, L. Xin, B. Fengwu, Appl. Energy 92, 65 (2012)

23. S.F. Hosseinizadeh, D.A.A. Rabienataj, F.L. Tan, J.M. Khodadadi, Int. J. Therm. Sci. 63, 55 (2013)

Cite this article as: Selvan Bellan, Alice Cordiviola, Stefano Barberis, Alberto Traverso, Jose Gonzalez-Aguilar, Manuel Romero, Numerical analysis of latent heat storage system with encapsulated phase change material in spherical capsules, Renew. Energy Environ. Sustain. 2, 3 (2017) 